\title{
On the Feeding Mechanisms of the Sei Whale (Balaenoptera borealis)
}

\author{
P. Brodie \\ Balaena Dynamics Ltd. Halifax. \\ Email: pbrodie@hfx.eastlink.ca \\ G. Vikingsson \\ Marine Research Institute, Reykjavik. \\ Email: gisli@hafro.is
}

Brodie, P., and G. Vikingsson. 2009. On the Feeding Mechanisms of the Sei Whale (Balaenoptera borealis). J. Northw. Atl. Fish. Sci., 42: 49-54. doi:10.2960/J.v42.m646

\begin{abstract}
The sei whale (Balaenoptera borealis) is a medium sized rorqual with basic features similar to the larger fin and blue (Balaenoptera sp.). While the sei whale has an expandable buccal pouch, it's capacity is substantially reduced by the length of the ventral grooves relative to fin and blue whales. The finer filtration capacity of the baleen allows predation on smaller prey spectra as a skim-feeder, with the option to engulf where prey are larger, or in greater densities. The sei whale exhibits some features of the mouth cross-section which are reminiscent of right whales (Eubalaena sp.) and there appears to be a difference in the degree of arch of the rostrum when comparing the sexes. The dynamics of skim-feeding and prey reaction are discussed.
\end{abstract}

Keywords: baleen, Bernoulli, biomechanics, engulfing, rorquals, sei whale, skim-feeding

\section{Introduction}

The sei whale (Balaenoptera borealis) is better known for it's non-conformity with other rorquals, often unpredictable in terms of migratory patterns and duration on the feeding grounds (Nemoto and Kawamura, 1977; Vikingsson, 1990; COSEWIC 2003; Olsen et al., 2009). When available, sei whale quotas were often taken over a brief period in Nova Scotia and Iceland. Sei whales were hunted for human consumption, and due to their smaller size, are processed quickly. Opportunities to examine a series of sei whales are rare, especially with ideal field conditions. We discuss their morphological differences which allow them to flourish in feeding areas considered suboptimal for other rorquals.

\section{Observations}

\section{Sei morphology relative to other rorquals}

The general morphology of the sei whale rostrum and baleen is shown in a series of photographs (Fig. $1 \mathrm{~A}-\mathrm{E})$ and in line drawings of the rostrum and baleen from fin, sei and bowhead whales (Fig. 2). Initially, sei appear to be a smaller scale fin whale. While they are generally described as slim, it is worth comparing their profile to fin whales, for example the sei is more robust with a thicker tail stock. The rostrum is noticeably arched, and appears to be more accentuated in the males when compared to females, an observation made when several sei heads were aligned on the cutting plan. The mandibles are curved as well, dipping slightly near the tip, matching the increased downward curvature of the rostrum. The obvious distinction is the shortness of the ventral grooves, restricting the capacity of the buccal cavity when expanded. The curved mandible is further enhanced by the raised lip which is higher relative to fin whales. The postmortem position of the lip of the sei whale slopes outward, away from the baleen rack. The entire lip extending along the mandible contains a series of specialized muscle bundles attached to the proximal surface of the mandible which increase in volume while arching outward across the upper surface of the mandible at a $45^{\circ}$ angle through the lip, diffusing into tendon at approximately half the height of the lip. Orientation of these muscles would function to retract the lips inward and against the baleen rack, similar to the fin whale (Brodie, 2001). This muscle arrangement would suggest that the initial outward angle of the lip would represent the relaxed or normal position, and drawn inward against the baleen rack in a voluntary action according to the filtering strategy of skimming or engulfing. The baleen is not as broad, nor as stiff as that of blue and fin, 


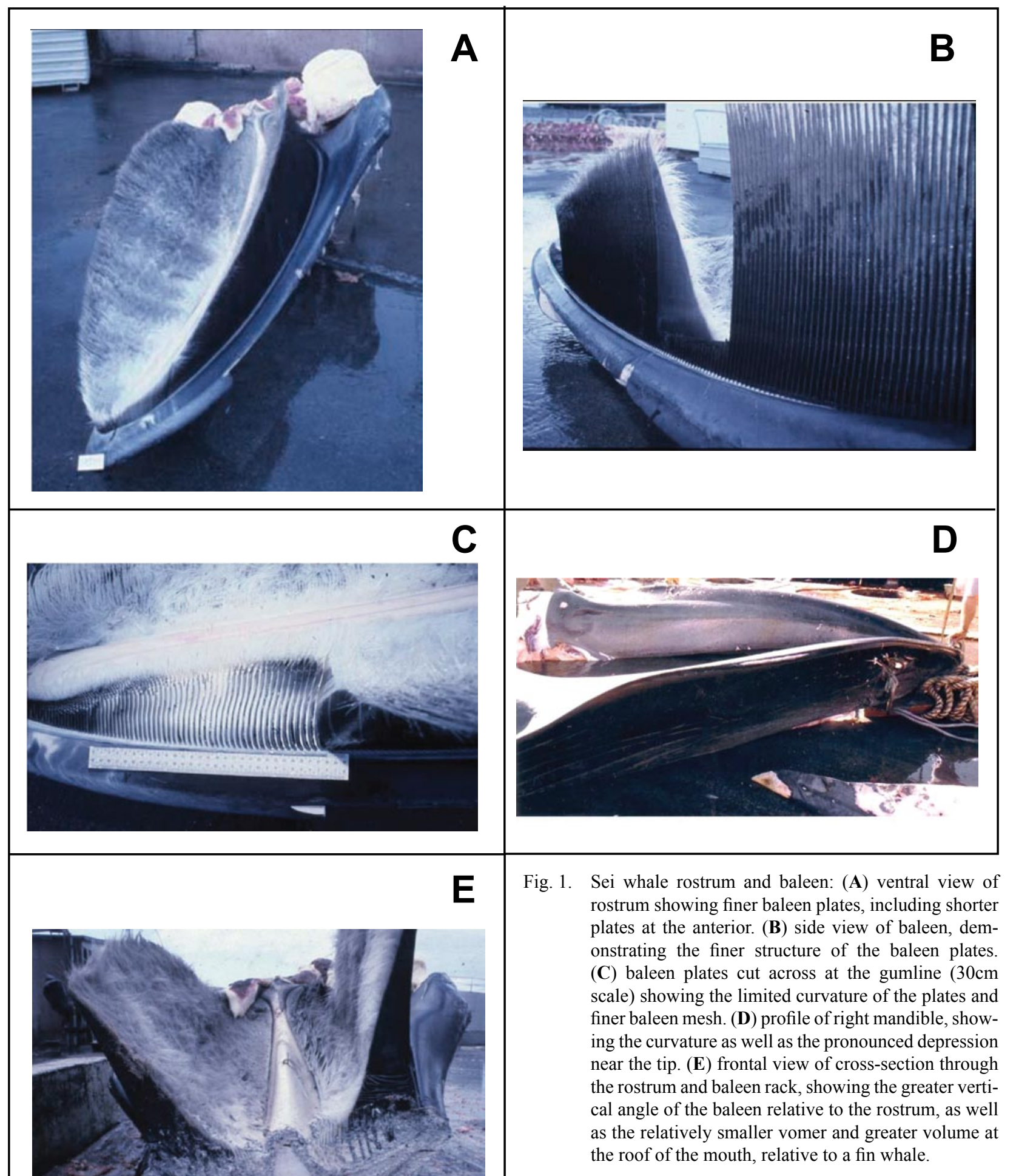




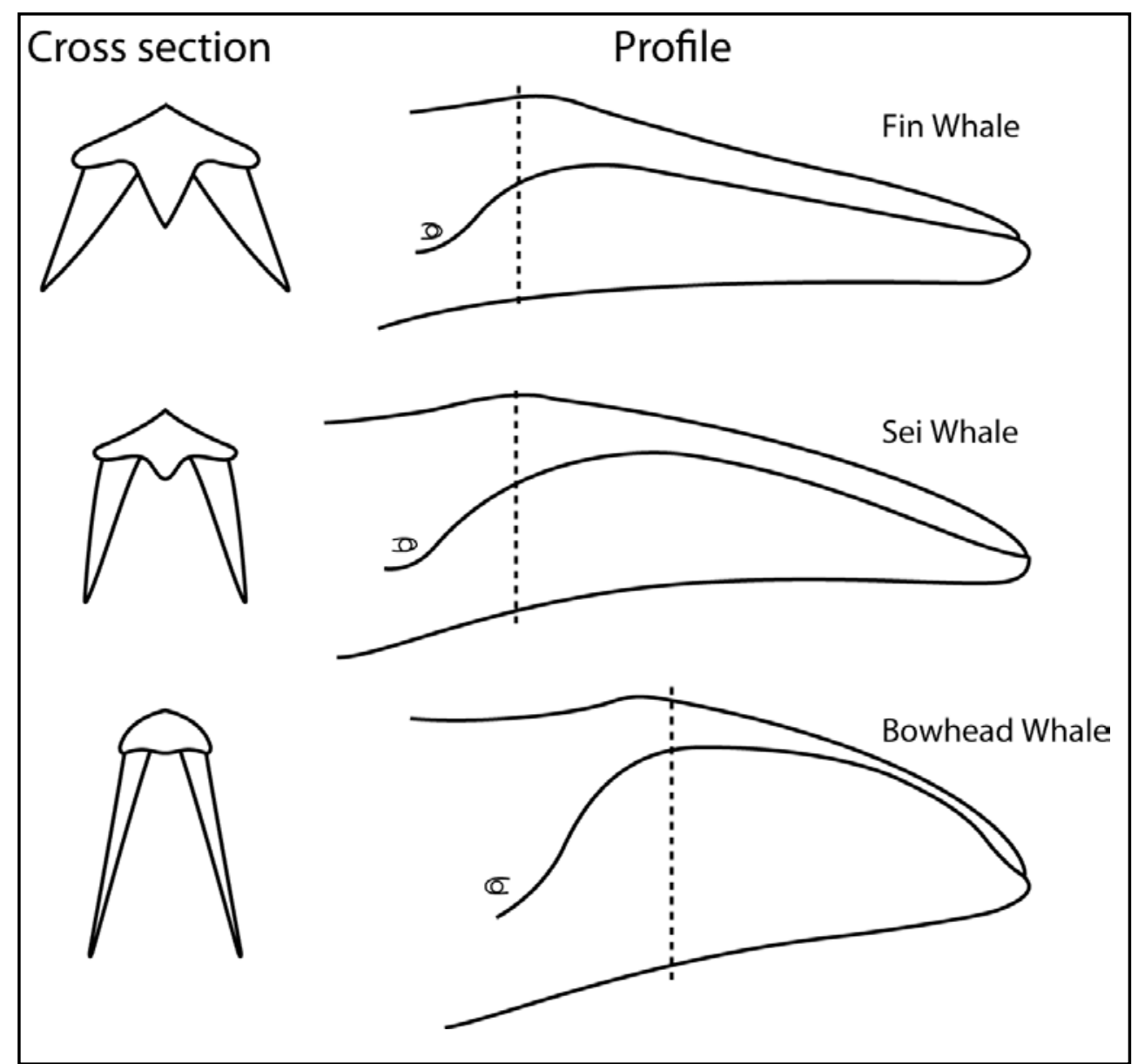

Fig. 2. Head profile and cross-section of rostrum and baleen. Fin, Sei and Bowhead illustrate the range of curvature of the rostrum, the sei being intermediate with a slightly arched rostrum and mandible, raised lip relative to the fin whale, with a greater curvature near the tip of the mandible. This modification would create an opening immediately under the shorter baleen plates and fibre bundles at the tip of the rostrum at partial gape. The rostrum cross-sections indicate the greater outward angle of the fin (and blue) baleen, as well as the greater prominence of the vomer relative to the sei. The sei has relatively longer baleen plates which hang at a more vertical angle from the rostrum, and which may allow the lips of the mandible to slide to a partial gape with less resistance against the baleen rack, similar to the Balaenids.

having a flexibility intermediate with right and bowhead. The structure of laminates and tubules is similar to fin and blue, however it is the change in proportions: longer, slightly thinner, less broad, smaller tubules, which contribute to the flexibility. Part of the flexibility is also due to the less complex cross-sectional curvature of sei baleen when compared to fin, blue and minke whales. A simple field observation which differentiates the sei whale from other rorquals, is that (observing at right angles to the rostrum) one is able to see daylight directly between the narrow baleen plates (as with bowhead and right), whereas the plates of other rorquals are broader with a greater curvature in cross-section. In rorquals, the baleen rack is comprised of an array of large baleen plates continuous in reduced size around the tip of the rostrum. By comparison, the smaller frontal baleen of the sei is not as long relative to that of a fin whale. These smaller frontal baleen plates and fibre bundles act as a barrier to forward escape by prey. The potential energy (elastic recovery) of the expanded buccal pouch creates positive pressure, forcing engulfed seawater through the baleen filter at a flow rate substantially lower than the engulfment phase, thus retaining the prey. This differentiates the rorquals from the Balaenidae (right and bowhead), in that rorquals filter out prey using a pressurized buccal pouch, once the water containing prey have been engulfed (Orton and Brodie, 1987; Goldbogen et al., 2006). Balaenids rely on venturi and Bernoulli effects 
(Lambertsen, 1983; Lambertsen et al., MS 1989; Werth, 2004) through a steady forward propulsion, generating continuous negative pressure within the mouth cavity, thus no requirement for a baleen barrier to prey at the tip of the rostrum. Prey are drawn to the inner surface fibre of the baleen filter of Balaenidae rather than forced against it by the elastic recovery of the buccal pouch, as with other rorquals. While it would seem that the large incoming volume entering the buccal pouch of rorquals increases the internal pressure, there is a simultaneous increasing external negative pressure posterior and external to the expanding pouch, acting to draw out the elastic pouch (Orton and Brodie, 1987). Through this action, formation of a detectable bow-wave is avoided. Once the buccal pouch is expanded to maximum capacity, the rostrum and mandibles are drawn together, with the forward escape restricted by the frontal baleen plates. Sei whales, in the skimming stance, must optimize the smaller mouth cross-section with the surface area of the exposed baleen at partial gape, which in turn, generates a Bernoulli effect, facilitated by external flow toward the negative pressure generated posterior to the expanded buccal pouch.

\section{Feeding on greater diversity over longer periods than other rorquals}

Any evolved feeding strategy is clearly economical. A shift, for example, to a skimming strategy, may have a lower or greater cost. However, the capacity to shift to a different feeding strategy allows a sei whale to feed in an area of lower densities of prey, possibly in regions which are warmer and less expensive in terms of daily maintenance (Brodie, 1975). This in turn, is reflected in the smaller body-size of sei whales, and reduced energy storage requirements.

\section{Morphological adaptations to skim feeding, compared to right and bowhead}

Detection of prey concentrations at close range, as well as the size of individual prey, may be reliant upon vibrissae on the rostrum as well as the mandible. This feedback might trigger either engulfing or a skimming stance. The degree of acuteness of the rostrum varies with the size of the rorqual: a broad rostrum in blue, to a sharp rostrum in minke (Arnold et al., 2005). This would shift the mass of the incoming seawater further back in the buccal cavity, and would reduce the force upon the most anterior part of the mandible and ventral grooves. In the sei whale, this design would allow greater control over the angle of gape while swimming forward in the skimming stance. A modification of the fibre size and orientation within the fibrous mass of the cranio-mandibular joint would be sufficient to maintain the jaw angle in the skimming stance, unless a threshold were reached, over-riding resistance in the joint, where the mandibles open to a full engulfing gape. This fibrous connective mass acts as an accumulator storing elastic energy to orient, and to assist in returning the mandibles to their normal position against the rostrum. The relative shearing forces within the fibrous complex at the base of the mandible may vary between species, the sei whale exhibiting greater shear when the jaws are partially open, thus having greater control over jaw gape. This was noted during postmortem tests of jaw loading in Iceland. The temporal muscle, sheathed in high tensile collagen, and attached to the angled coronoid process, acts as a fulcrum at full extension during engulfment (Brodie, 2001), generating a jaw rotation in concert with the craniomandibular joint. During the skimming stance, the temporal muscle and sheath would not be extended to the point where tension on the sheath bears the loading on the mandible. The temporal muscle exhibits no elastic modulus, shearing easily under laboratory tests, however it may provide sufficient tension in contraction to maintain assist in maintaining the partial jaw gape at slower speeds. As well, the lips of the mandible could exert additional force against the baleen rack, to further control the angle of gape. The ability of the sei whale to use either engulfing or skimming actions is evidence that both strategies provide net benefits, total energy input is exceeded by the energy gained, fundamental to the natural process of selective pressure.

\section{Simulated robust body profile by sei buccal expansion}

When the sei whale allows expansion of the buccal pouch, yet continues to swim in the skimming stance, the frontal half of the animal mimics the robust thorax of right and bowhead. In the sei, this would seem to result in a fine balance between the positive pressure of the seawater entering the buccal cavity, and the negative pressure immediately posterior to the expanded ventral grooves. With a square relationship between drag and velocity, the animal is able to maintain a balance between avoiding generation of a bow-wave, the venturi effect of water flowing between the baleen plates, combined with the Bernoulli effect from water flow along the outside if the baleen rack. Here, the vibrissae on the rostrum and mandibles may play a role in feedback, maintaining this critical balance. While all baleen in mysticetes appears to be similarly configured, the baleen of some rorquals seems less reliant on the Bernoulli principle than others, such as the sei. Essentially, a fin or blue whale uses the inertial energy to expand the buccal pouch, then uses the elastic recovery to force the seawater through the baleen 
rack at a flow rate which is lower than that during engulfment. The pressure drop relative to the flow rate, which is a fraction of the engulfing rate, causes the prey to be retained. During the skimming action of a sei whale, water is drawn through the baleen rack (rather than forced) by the negative pressure and velocity differences of the flow past the outside of the rack as a result of the negative pressure generated anterior to the expanded buccal pouch.

\section{Forces related to jaw opening}

Postmortem tests of the forces required to open the jaws of fin and sei resulted in estimates of $1900 \mathrm{~kg}$ (circa 19000 newtons) for a $19 \mathrm{~m}$ fin whale and 1000 $\mathrm{kg}$ (circa 10000 newtons) for a $15 \mathrm{~m}$ sei whale. It is interesting that, while there was additional force required to pull the mandibles over the outward projecting baleen rack of the fin (Fig. 2), this was not so apparent with the sei, however there was a noticeable resistance as the sei jaw was pulled to a partial gape of circa $35^{\circ}$ angle. It was suggested that fin whales may advance their lower jaw prior to engulfing, an action that would free the mandibles from the outward angle of the baleen rack. Once the buccal pouch is expanded, the base of the mandibles is forced apart, the rostrum is returned to position for the evacuation process (Brodie, 2001). The reduced scale of the feeding mechanism of sei whales, relative to massive engulfment by fin and blue, suggests that the sei may require a greater swimming velocity to activate the engulfing mechanism. The same argument can be extended to the minke whale, often observed to burst at the surface during a rush to engulf shoals of fish, an action related to the square of velocity (Arnold et al., 2005).

\section{Fluke control surfaces}

A notable feature of bowhead and right whales is the very large tail flukes, the largest of all cetaceans. Both appear to move their flukes at a slower rate than the rorquals, maintaining a low speed when skim-feeding. By contrast, the sei whale has flukes that are similar to blue, fin, minke and Bryde's (Bose and Lien, 1989). The larger flukes provide control and stability, propelling the robust-bodied skim-feeders as they feed. The sei whale, with the smaller flukes, appears to stroke with greater frequency to propel the expanded buccal pouch, during skim-feeding. Stability control may be achieved by the longer extension of the tail stock, thus extending the control surface for greater effectiveness. The pectoral flippers are not large, in terms of a planing surface when compared to right and bowhead, however this may be compensated for by the extended tail control surface.

\section{Acknowledgements}

We thank Kristjan Loftsson, Director of Hvalur H F, and his staff for their interest and cooperation over many seasons. Countless scientists have benefited from access to fresh material in a safe working environment, with skilled workers and laboratory facilities. In the 1960s K. Karlsen kindly provided access to the whaling facilities in Nova Scotia. Francis Kelly of Bedford Institute of Oceanography, Technographics Unit, put the professional touch to the illustrations.

\section{References}

ARNOLD, P. W., R. A. BIRTLES, S. SOBTZICK, M. MATTHEWS, and A. DUNSTAN. 2005. Gulping behaviour in rorqual whales: underwater observations and functional interpretation. Mem. Queensl. Mus., 51: 309-332.

BOSE, N., and J. LIEN. 1989. Propulsion of a fin whale ( $\mathrm{Ba}$ laenoptera physalus): Why the Fin Whale is a fast swimmer. Proc. Roy. Soc. B, 237 (1287): 175-200. doi:10.1098/ rspb.1989.0043

BRODIE, P. F. 1975. Cetacean energetics, an overview of intraspecific size variation. Ecology, 56: 152-161. doi: $10.2307 / 1935307$

2001. Feeding mechanics of rorquals (Balaenoptera sp.). In: Secondary Adaptation of Tetrapods to Life in Water. J.-M. Mazin and V. de Buffrénil (eds.). Verlag dr. Friedrich Pfeil, Munchen, Germany, p. 345-352

COSEWIC. 2003. Assessment and Status Report on the Sei Whale (Balaenoptera borealis). Pacific and Atlantic Population in Canada. COSEWIC, Ottawa, Canada, p. vii + 27. http://dsp-psd.pwgsc.gc.ca/Collection/CW69-14-3352003E.pdf

GOLDBOGEN, J. A., J. CALAMBOKIDIS, R. E. SHADWICK, E. M. OLESON, M. A. McDONALD, and J. A. HILDEBRAND. 2006. Kinematics of foraging dives and lunge-feeding in fin whales. J. Exp. Biol., 209: 1231-1244. doi: $10.1242 /$ jeb.02135

LAMBERTSEN, R. H. 1983. Internal mechanisms of rorqual feeding. J. Mammal. 64: 76-88. doi:10.2307/1380752

LAMBERTSEN, R. H., R. J. HINTZ, W. C. LANCASTER, A. HIRONS, K. J. KREITON and C. MOOR. MS 1989. Characterization of the functional morphology of the mouth of the bowhead whale, Balaena mysticetus, with special emphasis on the feeding and filtration mechanisms. Report to the Department of Wildlife Management, North Slope Borough, Barrow, AK, USA, from Ecosystems, Inc., Institute for Environmental Medicine, University of Pennsylvania Medical Center, Philadelphia, PA 19104, p. 134.

NEMOTO, T., and A. KAWAMURA. 1977. Characteristics of food habits and distribution of baleen whales with special reference to the abundance of North Pacific Sei and Brydes whales. Report of the International Whaling Commission (Special Issue). 1: 80-87. 
OLSEN, E., BUDGELL, E. HEAD, L. KLEIVANE, L. NØTTESTAD, R. PRIETO, A. M. SILVA, H. SKOV, G. VIKKINGSSON, G. WARING, and N. ØIEN. The feeding migration of a sei whale in the North Atlantic. Aquatic Mammals, 35: 313-318.

ORTON, L., and P. BRODIE. 1987. Engulfing mechanics of fin whales. Can. J. Zool., 65: 2898-2907. doi:10.1139/ z87-440

VIKINGSSON, G. A. 1990. Energetic Studies on Fin and Sei Whales Caught off Iceland. SC/41/Ba6, Rept. Int. Whal. Commn., 40: 365-373.

WERTH, A. J. 2004 Models of hydrodynamic flow in the bowhead whale filter feeding apparatus. J. Exp. Biol., 207: 3596-3580. doi:10.1242/jeb.01202 